\title{
Activating NOTCH4 Mutation
}

National Cancer Institute

\section{Source}

National Cancer Institute. Activating NOT CH4 Mutation. NCI Thesaurus. Code C158062.

A change in the nucleotide sequence of the NOTCH4 gene that that results in constitutive activation of both neurogenic locus notch homolog protein 4 and its downstream signaling pathways. 